\begin{tabular}{|c|c|}
\hline \multirow{3}{*}{ 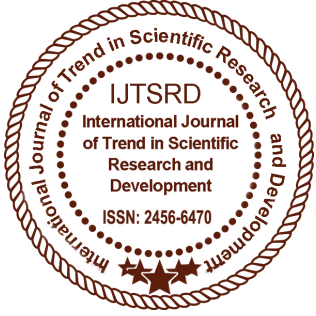 } & $\begin{array}{l}\text { International Journal of Trend in Scientific } \\
\text { Research and Development (IJTSRD) }\end{array}$ \\
\hline & International Open Access Journal \\
\hline & ISSN No: 2456 - 6470 | www.ijtsrd.com | Volume - 2 | Issue - 5 \\
\hline
\end{tabular}

\title{
Revolutionize Withering Process and Technology for World Tea Industry
}

\author{
I Jacob John \\ Jacobs Industry \& Creations, New Delhi, India
}

\begin{abstract}
The role of Withering is described as the changes that occur in the green leaf from the moment it is plucked from the bush to the time of maceration. As tea manufacturing technology evolves, the method and value of withering on black tea quality appears to be changing. In the production of flavour teas where tea is rolled, withering is considered vital for quality.
\end{abstract}

Withering is the first stage of manufacturing process. Unwithered leafs is difficult to ferment and dry.

Withering can be used as a means of storing the green leaf when leaf is very abundant or when it is short so that manufacturing can be effectively managed.

Withering Methods - Normal wither using Withering Troughs, Enclosed Withering Trough House to Twostage wither. From Age old inventions to the latest are Maceration machines operate most effectively within listed below: fairly narrow leaf moisture range.

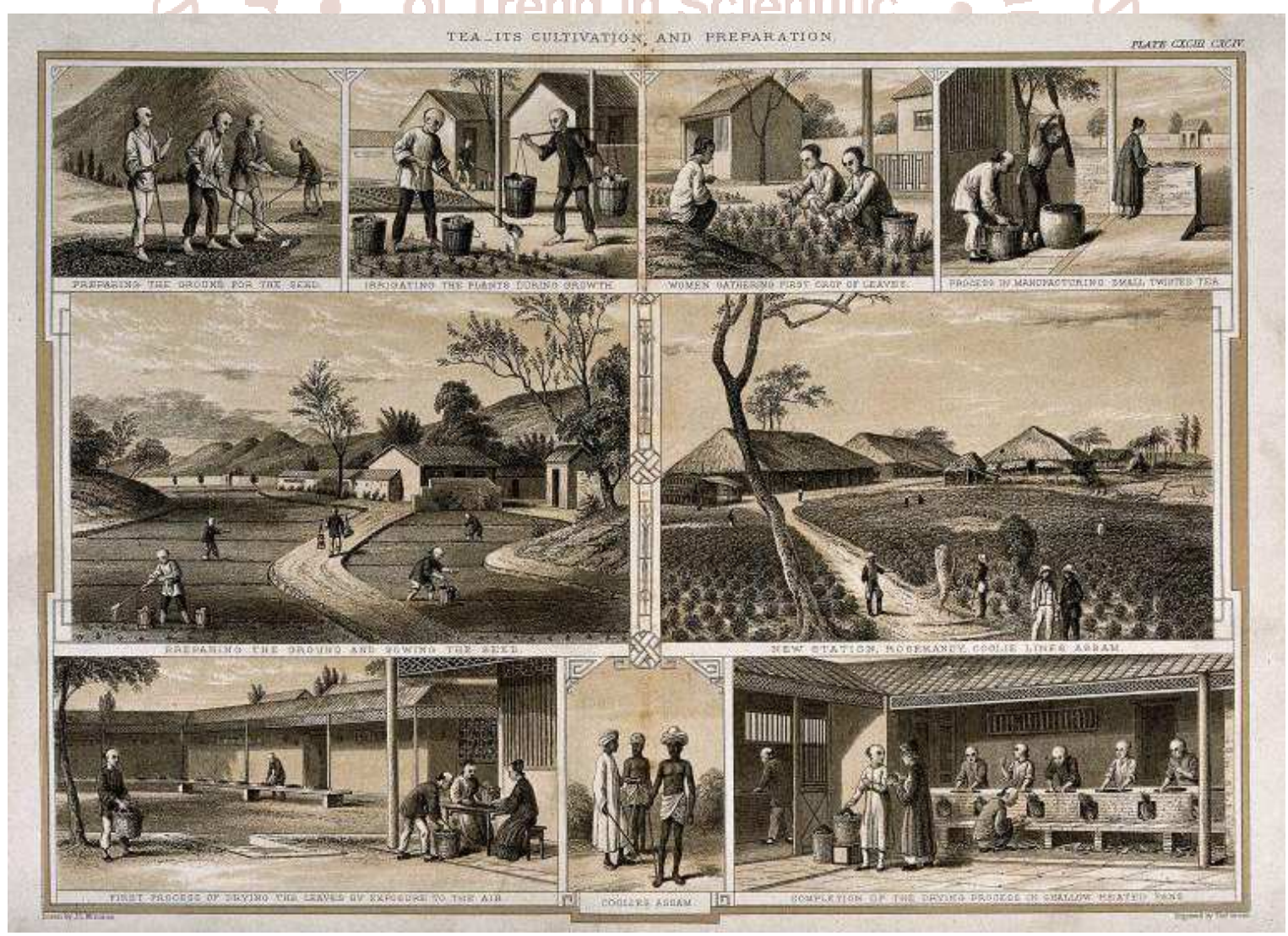

An 1890 British engraving showing tea cultivation and tea leaf processing. Withering is done on the floor. 


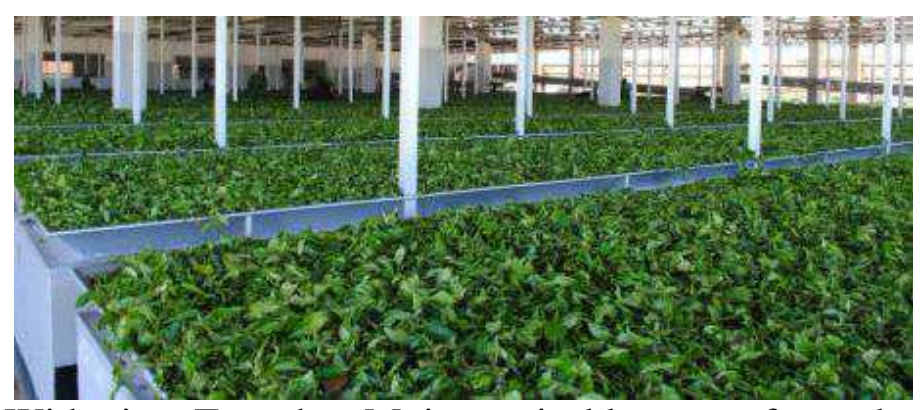

Withering Troughs: Moisture is blow out from the leaf by blowing air using axial fans and mixing of heat We see in many Indian tea factories especially south India withering troughs houses are built around the heater area so that they could benefit from its heat wave to increase the temperature of air blow into the troughs.

In areas where the heat is intense or proximity to the hearts, dampers are used to have a controlled mixing of heat with the ambient air that flows through the axial fans.

In larger factories (usually found in Africa and elsewhere) who use boilers for generation of heat for drying, a low pressure line is drawn to withering house and use heat exchangers placed in front of axial fans. This also does the job of warming the air that goes into the troughs. There are different designs and forms adopted viz. single radiator and a centrifugal fan draws hot air and ducted to each trough fan or each trough to have its own heat exchanger.

Withering Trough as the withering equipment has become very popular and used in all the tea growing countries. We also see an enclosed type withering system, the enclosed type has outweighed in merits over open type trough, many tea groups adopted this as their manufacturing standard. (picture not provided as this may infringe their rights if any on their design)

In 1970s mechanisation in withering trough feeding was tried at Sotik Tea, in Kenya, developed a machine for loading of tea leafs into withering trough. It's a electrically operated and hydraulically driven wheeled loading machine on rails to feed the leafs on to the trough bed.

In the later years in 1990s towards end of century tea industry has seen working on the two stage withering system and has developed machines in the parts of Africa

Lead design is from Unilever where they adopted jumbo troughs to hold leaf and a multilayer perforated mesh conveyor belt driven system in a continuous stage to remove moisture.

Later new design adopted multilayer perforated mesh conveyor one over other in a chamber was developed in Malawi and Kenya. (Picture not provided as this may infringe their rights if any on their design)

China's too have this system since many years where it has 5 to 6 layers of Stainless Steel Mesh Conveyor decked one over other. This design is different over the ones in Africa as the warm air is passed through individual layer to pass thru the leaf and disperses to atmosphere. (Picture not provided as this may infringe their rights if any on their design)

\section{Technology Discovery}

In the previous subject we have seen various evolutions happened over the years. Ideas were implemented and tried. Interestingly last one century seen developments in Kenya in this section.

Withering is the largest energy consumer in the processing of tea. Even cost of infrastructure is major for withering.

Here we are listing a scientific analysis on how the moisture is reduced in Tea Leafs in all the existing systems.

In most of the systems adopted - some were about automation to reduce labour (save costs), some revolved around the study on less human interference to reduce damage to leaf to improve on made tea quality, to more adyanced mechanisation to save on space, controlled moisture removal and throughput. Different designs but with same working principle.

We can summarise that ambient air \& heat are the key components that is induced in a certain manner to achieve removal of moisture to the desired moisture level in the withering process. This thought process has never been questioned as it is fairly understood that warm air dries leafs or any wet commodity.

We deeply study here, what happens when heat is induced into the air:

A. We know when air is heated - it increases in volume and increase in pressure or both, depending on the conditions. Here our key ingredient is "air when heated increases in volume" 
B. We also know atmospheric air contains humidity and percentage of humidity depends on the various factors like altitude, place etc. We measure it in the form of Relative Humidity, also called as $\mathrm{RH} \%$.

Variations in these two key components (heat and humidity) in the atmospheric air are what I want to understand (an illustration on the RH\% variation vs heat).

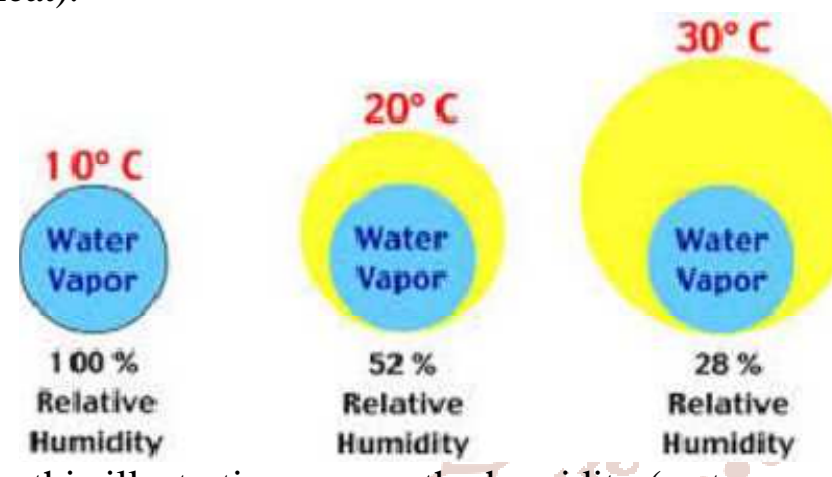

In this illustration we see the humidity (water vapour) component has not changed when the air is heated while its volume increased and percentage of $\mathrm{RH}$ has reduced.

The principle to remember is that warm air can hold more water vapour than cold air.

Here we made a case that extra volume of air that has increased due to infusion of heat has created more room to absorb water vapour. When the warm air is passed through the withering troughs that extra volume of air will start attracting the moisture from leafs and fill into itself. This exercise leads the leafs to wither by losing its moisture to the air blown through it.

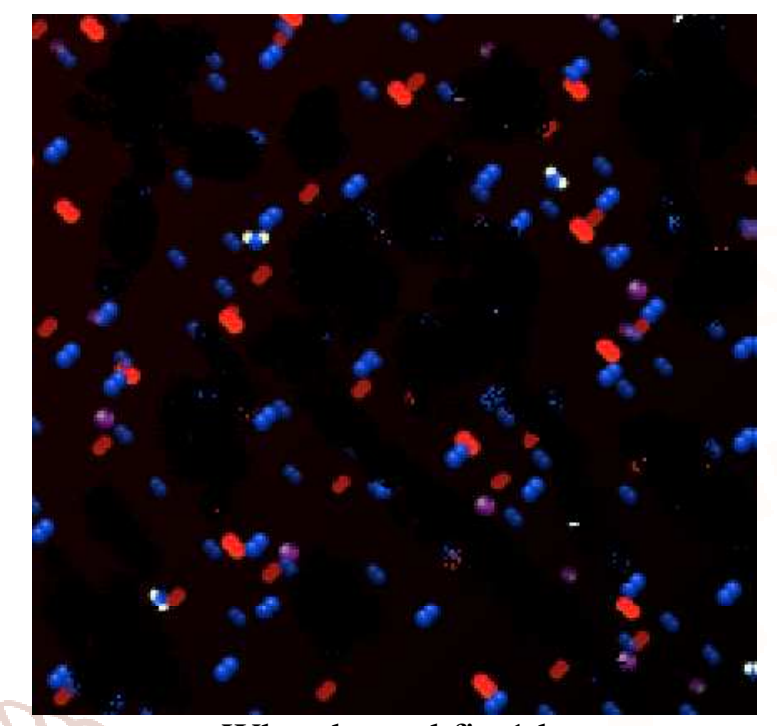

When heated fig 1.b

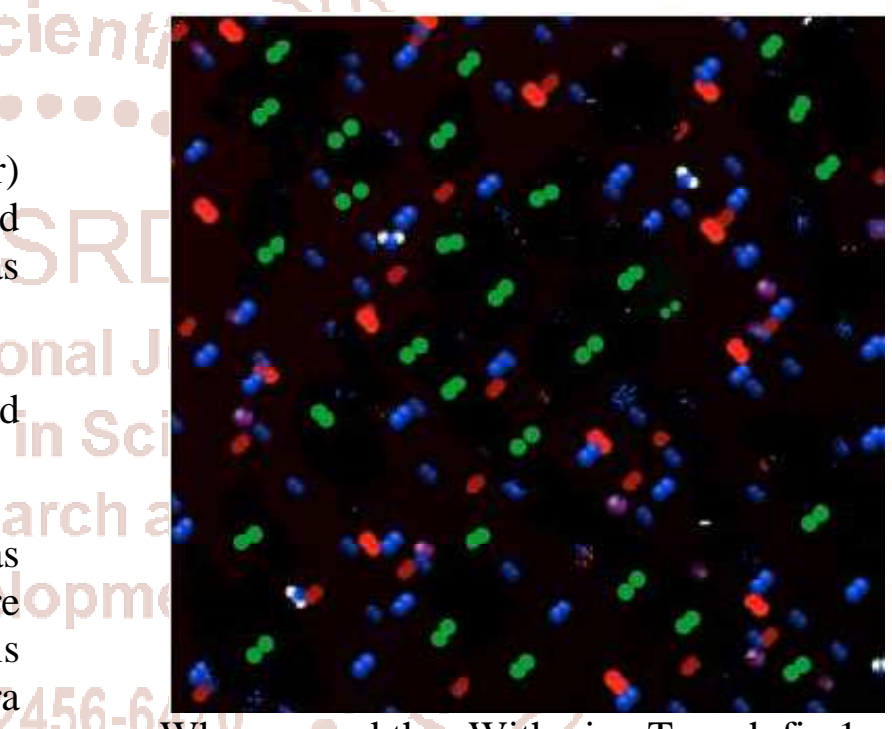

When passed thru Withering Trough fig 1.c

We can see the behaviour of air volume how it transferred from dense state to heated state and how the air carried the moisture from the leaf bed.

Let us see an illustration on air density before, during and after in the process of withering.

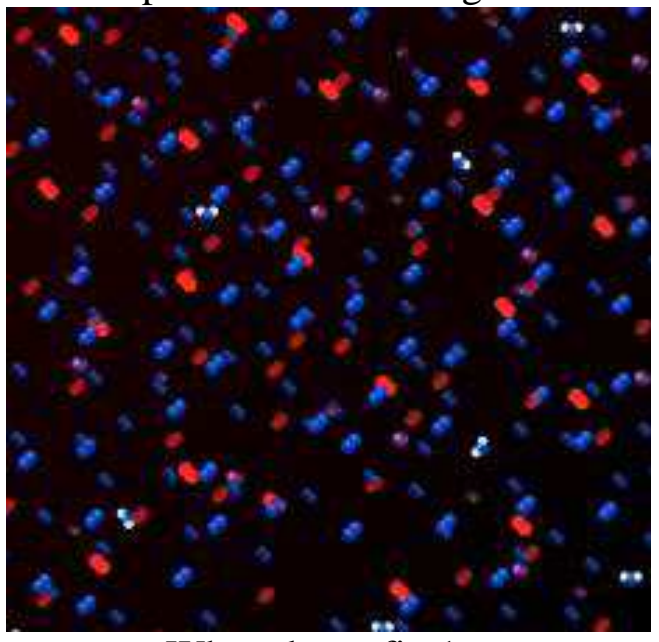

When dense fig 1.a
The density of air is the mass per unit volume of Earth's Atmosphere. Air density, like air pressure, decreases with increasing altitude. It also changes with variation in temperature or humidity.

We can visualise this concept as to how it is conducted. a) the efficiency of the transfer of moisture depend on the time it takes to travel through the leaf bed b) the warm air (reduced $\mathrm{RH} \%$ as per the above illustration) is pushed into trough chamber and a back pressure is built to enable air to escape through leaf bed. We can see amount of power is used to build or maintain air pressure inside the trough. We can see the resultant air that passed through the trough in the above illustration. 
From the above illustration it is evident that the variation in $\mathrm{RH} \%$ carries away the moisture from the tea leaf.

All the developments in the withering system revolved around the "warm air" as the key ingredient for extraction of moisture from the leafs. It is this principle that is adopted in all the works or R\&D conducted in the withering section of the tea manufacturing.

NOW THE CASE: If the RH\% is the key to remove moisture from the tea leafs. We want to analyse if we can adopt other methods to achieve this medium at a lesser cost, optimum utilisation of the medium, savings on power and space. Also whether it can give a revolutionary machine design.

In the current withering process we see 3 areas 1.Heating, 2.Excess power for pressure, 3.Duration of transfer of moisture from leaf to air.

We can see system inefficiency in terms of energy loss and inability to control duration of moisture transfer.

In the above illustration, we can see the difference between figure 1.a \& figure 1.c while it has more space to occupy moisture/vapour post heating up the air, we see much space left due to inability to carry vapour as we do not have any method to control the duration of moisture transfer.

We know from "Abstracts in withering system" a system called Closed Withering House used by big weights of tea industry; they achieve better results owing to controlled inward and outwards air flow both below and over the troughs.

Instead of heating the air by using external means, to increase volume of air or to reduce $\mathrm{RH} \%$ per volume for blowing it through the leaf to carry away the moisture, we can adopt other methods to achieve same or higher level of reduction of $\mathrm{RH} \%$ in the given volume of air.

We have known other methods:

A. To reduce $\mathrm{RH} \%$ in the air / remove moisture

B. Control the flow of air through the leafs

This may also have advantage over the current principle where we are concentrating only on increasing the volume of air (reducing the $\mathrm{RH} \%$ ) to absorb more moisture / vapour. Also key ingredients i.e. Heat - to convert moisture to vapour.

We can conclude that withering needs:

A. Dry air - to carry vapour

B. Heat - to transfer moisture to vapour

C. Controlled Processing - time needed for moisture to transfer to vapour and complete absorption of vapour to dry air.

\section{DRY AIR}

Option: We can remove moisture in the AIR by compressing \& drying method.

The process of air compression concentrates atmospheric contents, including water vapour. This raises the dew point of the compressed air relative to free atmospheric air and leads to condensation. The condensed water can be removed. We also know how in the industrial compressed air plants, water is removed using condensate drains, membrane filters, refrigeration dryers etc.

\section{HEAT}

In the process of removing moisture from the compressed air, the heat generated in the compression loses when it passes through the refrigeration dryer. Same need to be heated using external heaters or routing it through the heat exchanger of the dryer to achieve the desired heat.

In the withering we need only WARM AIR. This can be easily achieved through utilising the heat exchanger of the Dryer.

\section{CONTROLLED PROCESSING}

Can use a Air Tight closed chamber with both air inlet and air outlet timed processing. Spent air will be damp air - to be removed through the air outlet and replaced with Dry Air from through the inlet. This cycle depends on the moisture content in leafs as well as desired reduction of moisture $\%$ in the leaf.

Leaf will respond to the inlet Warm Dry Air, allowing moisture to vaporise, saturating the dry air inside the chamber. Dry air will start accumulating RH\%.

Since the Air inlet and outlet is controlled through the timer, we can determine the time needed to achieve a maximum $\mathrm{RH} \%$. We can use a Hygrometer to 
measure the desired \% of $\mathrm{RH}$ inside the Chamber to control inlet and outlet of air.

Through this process we can achieve a desired result at a given time, at a given volume of air and temperature. We can have a complete control on all the three factors that is required to achieve withering.

In nutshell, I want to make an illustrative demonstration of air.
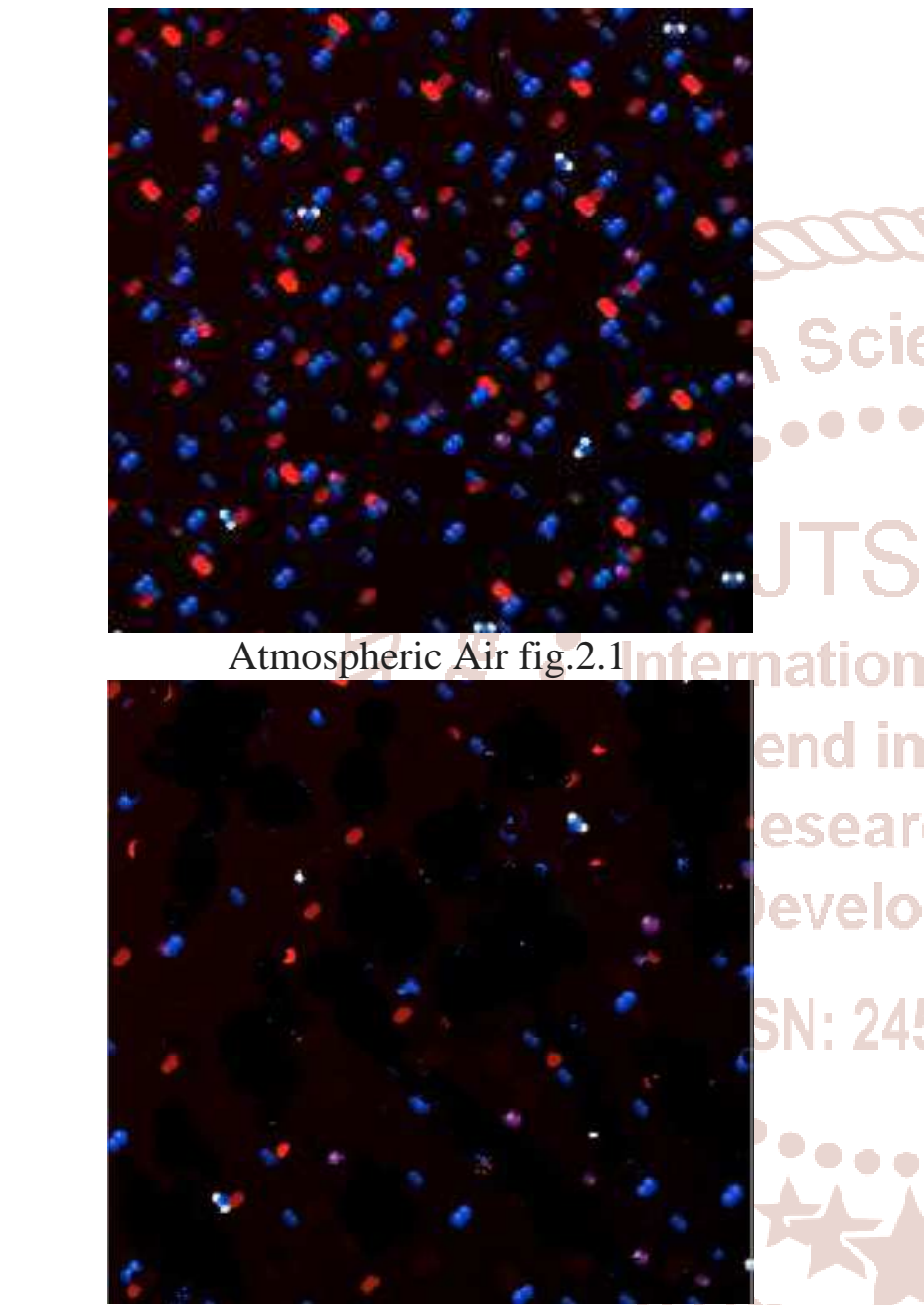

Treated Air after Compression \& Drying fig.2.2

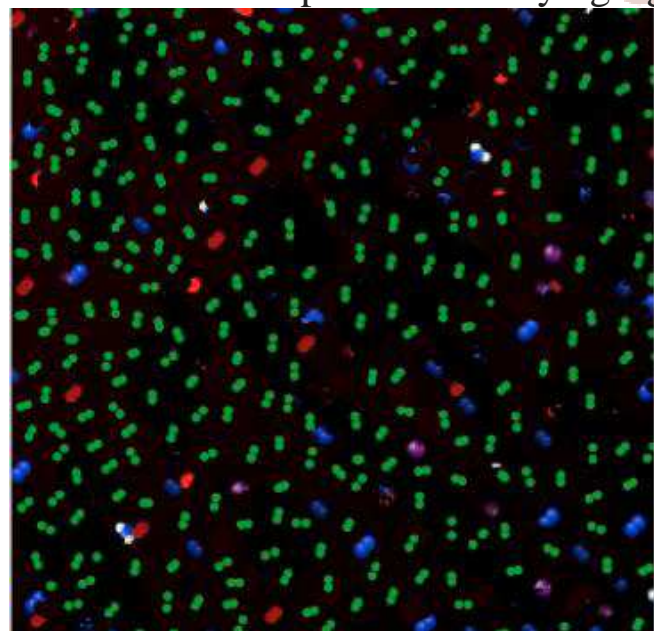

Air from Withering Chamber fig.2.3
A popular quote by Peter Drucker - You can't manage what you can't measure. If you can't measure then, you can't improve it.

By this method I am of opinion that with the controlled processing we can achieve

A. Desired level of Withering

B. Optimum required power needed for withering

C. Small foot print of the equipment design

D. Energy cost would also be lower

This Withering system would have these components

\begin{tabular}{|l|l|l|}
\hline \multicolumn{2}{|c|}{ Compressed Air Plant } & Leaf Processing plant \\
\hline a. & Compressor & a. Vacuum chamber \\
\hline b. & Air Receiver & $\begin{array}{l}\text { b. Inner mechanism to } \\
\text { tumble leaf }\end{array}$ \\
\hline c. & Filtration System & c. Leaf feeder \\
\hline d. & Refrigeration Dryer & d. Leaf discharge \\
\hline e. & $\begin{array}{l}\text { Pre and Post filtration } \\
\text { system }\end{array}$ & e. Hygrometer \\
\hline f. & Dry Air Receiver & f. Vacuum pump \\
\hline g. & $\begin{array}{l}\text { Condensate drain } \\
\text { system lall }\end{array}$ & g. Valve \\
\hline h. & Piping & h. Controller \\
\hline i. & $\begin{array}{l}\text { Common control panel to monitor the air } \\
\text { consumption, flow rate, timing and generation } \\
\text { of dry air }\end{array}$ \\
\hline j. & $\begin{array}{l}\text { Continuous mechanisation for leaf feeding and } \\
\text { discharge }\end{array}$ \\
\hline k. & Throughput controlling \\
\hline
\end{tabular}

This is in conceptual stage a thorough study and trials with miniature system is needed to study the behaviour on following areas:

A. Behaviour of leaf when treated with dry air and temperature to vaporise moisture in controlled environment

B. Rate at which the withering will be achieved

C. Consistency on end results in different climatic conditions, quantities, moisture percentages

D. Estimate volume of dry air needed to wither $x$ amount of leaf

Running cost will be ascertained from these factors

A. Rate \& Volume of Dry air required to wither $\mathrm{x}$ value of leaf

B. Power required to generate that Dry air per Minute / per batch / per weight

C. Power required for Leaf processing plant based on its design 


\section{Technology Design}

System will look something like this:

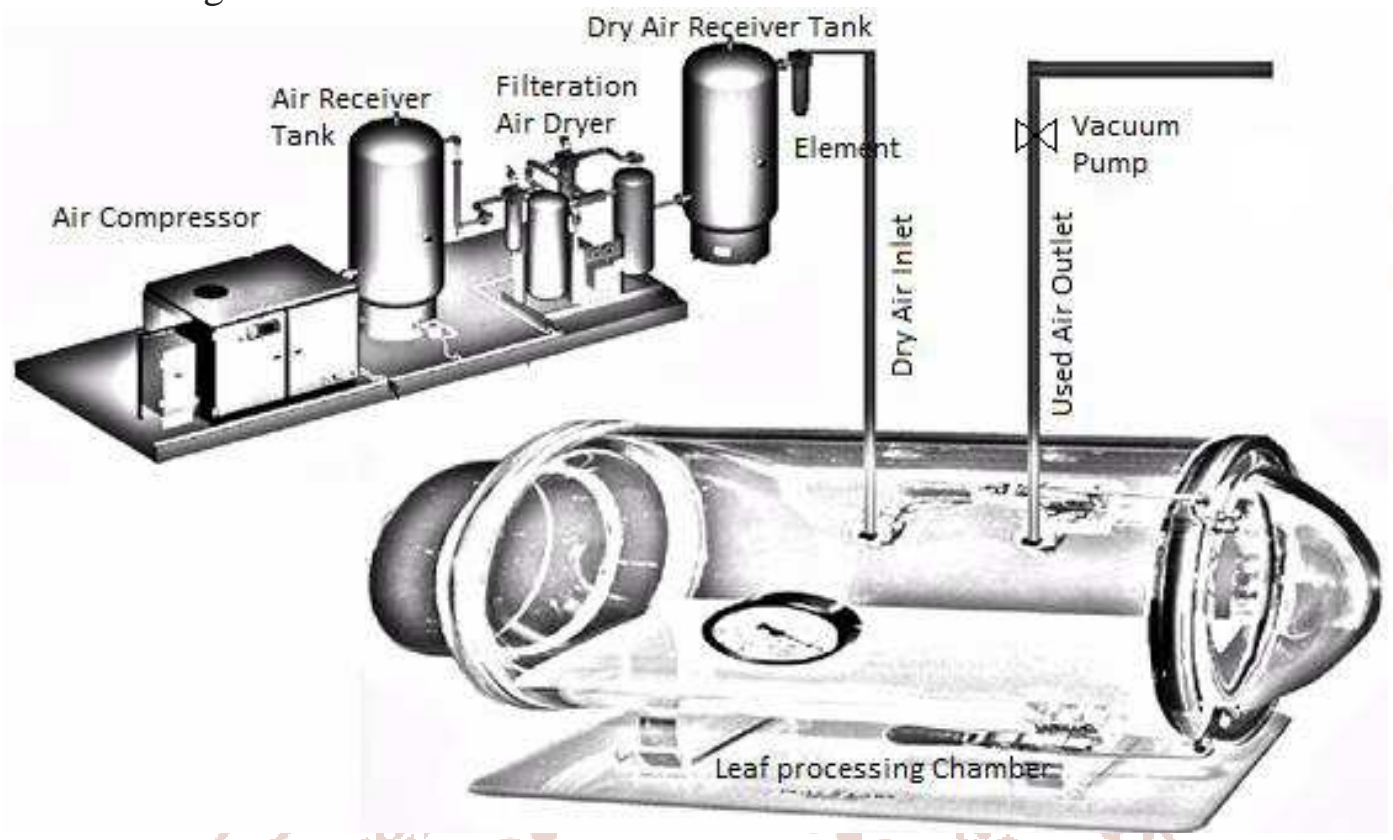

A final design can be achieved only when practical discharge of leafs.

research is carried out.

Above picture is only a basic concept based on

Feeding and Discharge mechanism can be conceived norking principle.

based on the design that would evolve on the leaf processing chamber.

Note: this just one concept, more on anvil

Through put - can be measured based on the outcome of leaf response and time taken to wither.

Sizing of the compressor depend on outcome of research.

Complete automation is possible in controlling air, drying, air inlet and outlet timing, feeding and
Author: I. J. JOHN

About the author:

I had a brief stint in tea industry in my life that helped to interact with people, factories in India, China and Africa. Legacy of World of Tea, has given me a lasting experience be it Tea Manufacture, Tea Estates and the life style surrounding TEA.

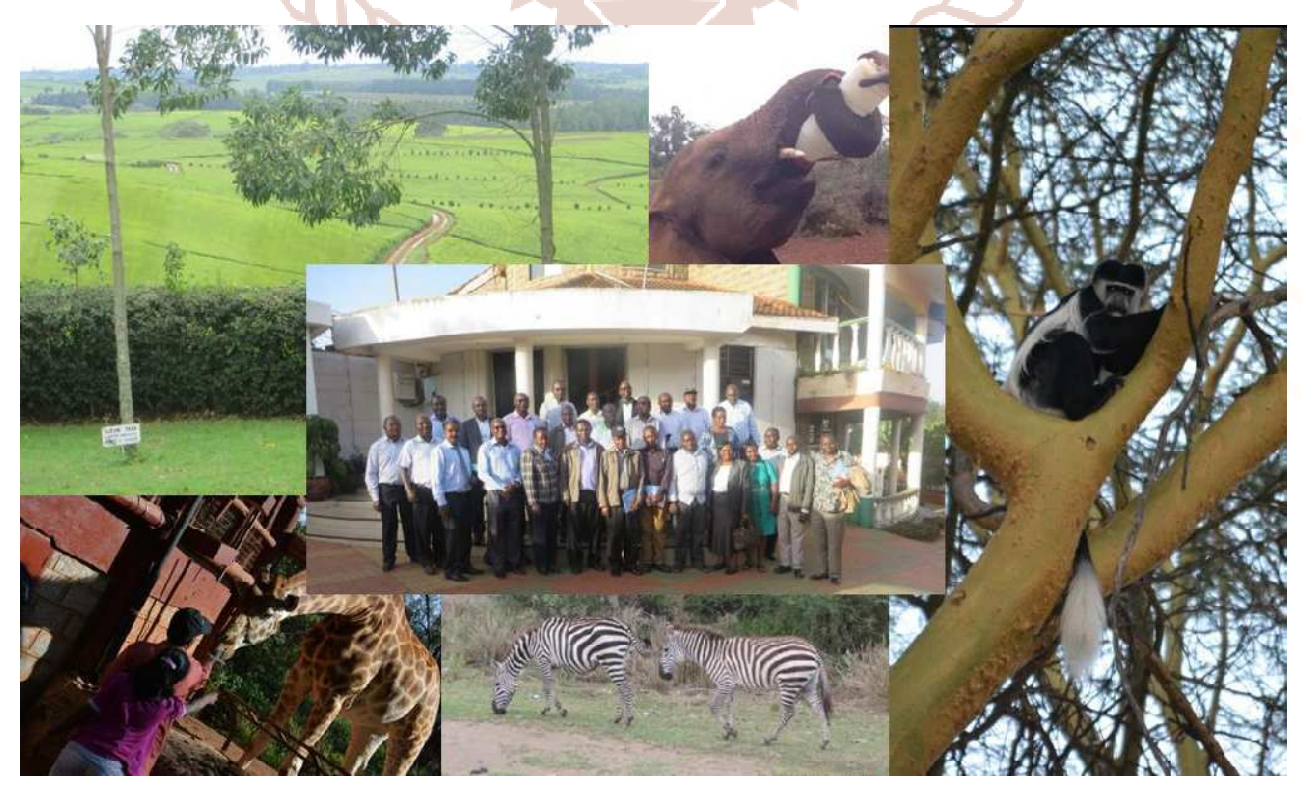


Disclaimer: In accessing, browsing and using the pages of Jacobs.net.in (hereinafter referred to as "the Site") the user (hereinafter referred to as "the User") accepts, without limitation or qualification, the terms and conditions herein.

The contents and works of this article reflect authors imagination only. Author does not warrant the accuracy or completeness of the information, text, graphics, links for other items contained in this website.

No license or right to use any of the aforesaid contents of this site is granted to the user; no portion of this site may be reproduced, copied, published or used in any form or by any means for commercial purposes without prior written permission from the author. Users are permitted to view, print or download extracts from this site. Authors do not guarantee of its accuracy, completeness, reliability, usability of the content and does not carry any liability. The respective user is therefore generally obliged to professionally check or to have professionally checked the suitability of all content for its intended use.

The information contained on this site is authored or complied by the author sources, unless otherwise specified. Author reserves the right to change the contents, function or operation of the site at any time without prior notice.

The contents of this site have been prepared for general informational purposes and do not constitute an offer to sell or solicitation to invest. However, if a user becomes aware of a copyright infringement, author asks the user for notification. Upon notification of such violations if any, author will remove the content immediately.

For any further information or clarifications, the user should go to the "Contacts" page of this site.

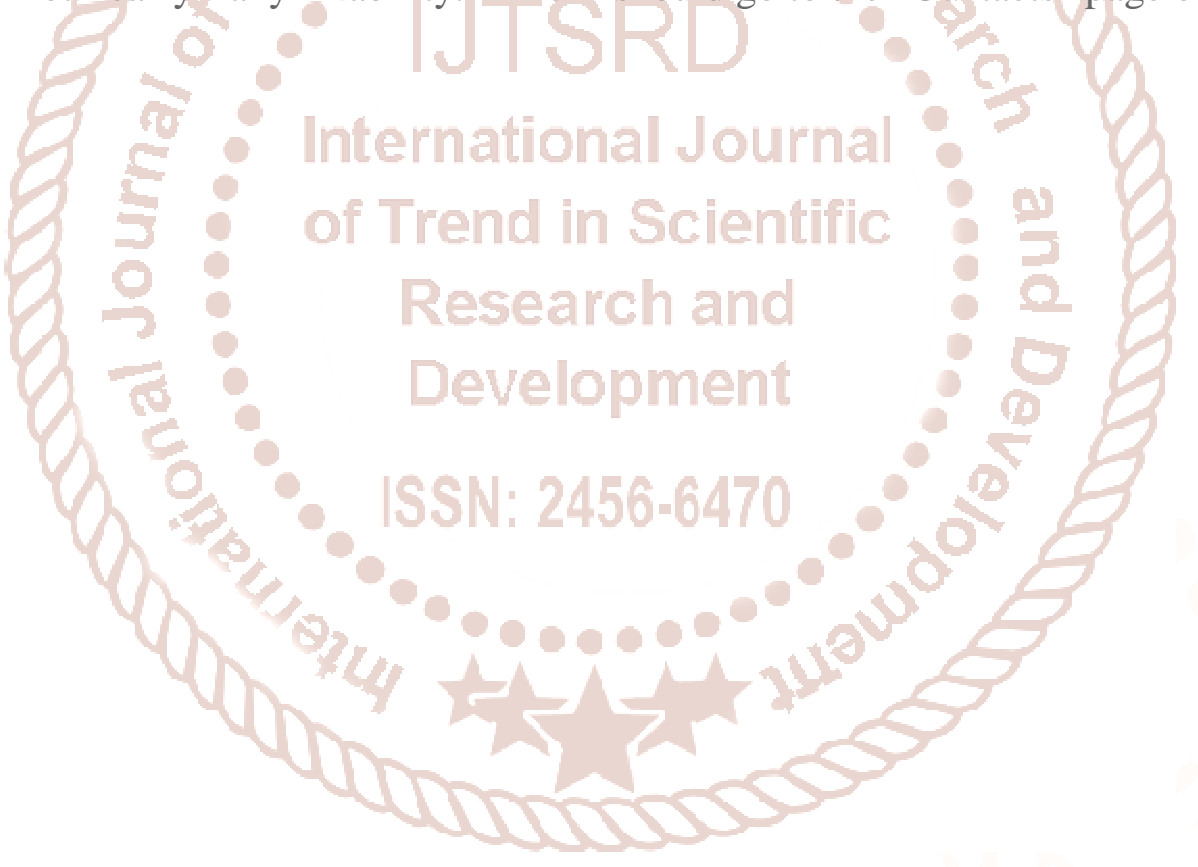

